\title{
Francisco Proença Garcia, Análise global de uma guerra. Moçam-bique 1964-1974, Lisbonne, Prefácio, 2003, 408 p., bibl., ISBN : 972-8563-97-3, préface de Adriano Moreira, postface de Fernando Amara Monteiro («Estudos e Documentos/História militar »).
}

Cet ouvrage est fondé sur un gros travail au sein d'archives militaires auxquelles l'auteur a eu accès (Arquivo da Divisão de informação do EMGFA, Arquivo histórico militar, Arquivo do serviço de documentação e história militar), la recherche dans les archives civiles semblant plus faibles (Arquivo Oliveira Salazar et Histórico-Diplomático, certes, mais pas les archives de la PIDE-DGS ou des SCGIM). Il s'agit d'une vision très classiquement «campiste », parfois événementielle, et descriptive : la guerre coloniale n'est qu'un succédané de la guerre froide, etc. L'« analyse globale » annoncée devient alors une fresque panoramique, très " portugaise ", de la guerre - même s'il n'y a pas d'outrance anti-Frelimo. L'ouvrage regorge cependant de données (y compris dans la postface de Amaro Monteiro, qui sait de quoi il parle), qui pourront être utiles aux historiens.

En fait, c'est une vision strictement militaire qui nous est livrée : les données d'histoire des peuples africains, du Frelimo, sont des plus succinctes et vues uniquement sous l'angle de l'action psychologique (APSIC) portugaise. On aurait aimé, par exemple, une étude sur les chefs traditionnels face à la guerre, sur le type de recrutement d'Africains par l'armée coloniale, sur les aldeamentos (statistiques, situations, typologie). On aurait aimé aussi une analyse politique de la population blanche. On comprend que l'auteur n'ait pas eu comme objectif d'écrire une histoire globale de la guerre - il n'y a pas d'étude des grandes campagnes militaires, telles le Nœud Gordien (traité en une seule page, p. 192), ni de la contestation montante parmi les militaires. Cependant, il me semble que les grandes forces en présence devaient être historiquement analysées: pourquoi n’y a-t-il pas eu lutte armée en Zambézia? est-ce uniquement le facteur ethnique? n’y a-t-il pas une histoire particulière de la colonisation dans cette région? pourquoi les Macondes ont-ils été le fer de lance de la lutte? est-ce uniquement à cause de leur position frontalière? qu'est-ce qui explique l'unicité du Frelimo? Etc.

L'ouvrage contient trois chapitres. Le premier chapitre porte sur le jeu des grandes puissances en Afrique, du Congrès de Berlin à l'anticolonialisme de l'Onu, avec deux sous-chapitres sur la situation interne: l'un sur les "linhas de fronteira $e$ substratos sócio-religioso anteriores e remanescentes » (pp. 37-51) qui utilisent, sans les discuter, des concepts datés du genre «tribus" pour classifier les peuples africains; l'autre sur «A política interna portuguesa e as ópticas quando do Ultramar» (pp. 72-104) qui contient quelques données utiles sur l'opposition civile et les hésitations des militaires - mais ne contient presque rien sur le PCP. Le deuxième chapitre s'intitule audacieusement "A conflitualidade global permanente " et présente l'anticolonialisme mozambicain comme reflet de la subversion mondiale, et la réponse portugaise sur les plans politique (très succinctement: aucune analyse de l'« étatisation » du Mozambique en 1970-72), militaire et de l'APSIC. Le troisième chapitre présente les peuples mozambicains dans leur rapport à la subversion, selon la classification ethnique des services d'information militaire eux-mêmes, et socioreligieuse.

Plus généralement, on constate une étrange absence de distance entre l'auteur et ses sources. Celles-ci - de quelques bords qu'elles soient, mais elles sont à $99 \%$ 
issues du côté portugais . . - - semblent dire « la vérité ». Ainsi, la population vivant dans les zones libérées du Frelimo est évaluée à 1800000 personnes «organisées en coopératives », parce que ce chiffre (p. 18 et note 815 p. 359) est dans un article de la presse... danoise, envoyé par l'Ambassade portugaise dans ce pays au ministère des Affaires étrangères et se retrouve donc aujourd'hui dans un dossier de l'Arquivo Histórico-Diplomático (en réalité, probablement 300000 personnes vivaient totalement en «zones libérées »). Ainsi, l'émeute de Mueda n'aurait rien à voir avec les Macondes du Tanganyika (p. 133 et note 523 p. 344) parce que le consul belge à Lourenço Marques, en un rapport fort mal renseigné capté par la PIDE, le dit, et dit aussi qu'il s'agissait en fait d'une révolte contre la culture forcée du coton. Or, il n'y avait aucune culture forcée du coton sur le planalto maconde et l'émeute eut lieu à l'occasion d'une réunion publique en présence d'un délégué maconde du Tanganyika lié aux associations de l'émigration mozambicaine dans ce pays. On trouvera des dizaines d'exemples similaires dans le livre. L'auteur ne discute pas ses sources, il les accumule simplement. De même, des erreurs de noms sont fidèlement reproduites, parce qu'elles figurent dans les archives (ainsi «John Marvem» au lieu de John Marcum, p. 350).

Cette très grande carence méthodologique dans l'ouvrage est pourtant à mettre en rapport avec un phénomène plutôt positif et que souligne Adriano Moreira dans sa préface: «Le nombre d'officiers des Forces armées qui cherchent la consécration de titres universitaires, s'accroît» (p. 15). Effectivement, l'auteur est militaire, diplômé en «sciences militaires » de l'Académie militaire en même temps qu'il est docteur en histoire contemporaine d'une université privée, l'Universidade Portucalense. On ne peut que se réjouir de cette «tendance universitaire » chez certains militaires, qu'ils aient connu ou non la guerre coloniale. Mais on peut aussi questionner leur encadrement et leur préparation pour devenir docteur en sciences sociales. Pourtant le Portugal, moderne et démocratique, a aujourd'hui d'excellents centres d'études africaines. Plutôt que de créer l'« université des Forces armées » souhaitée par Adriano Moreira, mieux vaudrait utiliser les outils excellents existant dans les universités publiques.

Mai 2005, Michel Gahen

\section{Álvaro Nóbrega, A luta pelo poder na Guiné-Bissau, Lisbonne, Univer- sidade técnica de Lisboa, Instituto superior de ciências sociais e políti- cas, 2003, 334 p., bibl., index, ISBN : 972-8726-19-8.}

Pourquoi publier une thèse de mestrado, que l'on peut comparer en France, selon les cas, aux anciennes thèses de troisième cycle ou aux mémoires de masters de cinquième année d'université ? La recherche ici présentée aurait en effet gagné à être approfondie en thèse de doctorat, si possible en élargissant le cadre institutionnel où l'auteur a "vécu ", à savoir l'ISCSP, à d'autres milieux académiques et scientifiques. La recherche menée a été importante, malgré des conditions difficiles. Les sources, cependant, restent lacunaires, des travaux considérables de langue anglaise ne semblant pas connues (livres de P. Chabal, R. Chilcote, J. Forrest, R. Lobban, L. Rüdebeck). Il en va de même pour ceux, moins nombreux, de 